\title{
Diagnostic de la mort dans le contexte de la transplantation
}

\section{Michelle Salathé}

lic. iur. MAE, Secrétaire générale adjointe de I'ASSM

1 Sous-commission: Prof. Dr méd. Jürg Steiger médecine interne, Bâle (Président) Prof. Dr méd. Claudio I. Bassetti, neurologie, Berne Prof. Dr méd. Bernhard Frey, médecine intensive pédiatrique, Zurich Prof. Dr méd. Christoph Haberthür, médecine intensive, Zurich PD Dr méd. Yvan Gasche, médecine intensive adultes, Genève Eva Ghanfili, NDS HF, soins intensifs, Lugano Prof. Dr méd. Samia Hurst, éthique, Genève Prof. Dr méd. Christian Kind, pédiatrie, Saint-Gal (Président CCE) Corinne Delalay-Marti, NDS HF, Sion lic. iur. Michelle Salathé, MAE, droit, Berne (ASSM) PD Dr méd. Urs Schwarz, neurologie, Zurich Et en tant qu'expert permanent: Marcel Monnier avocat, OFSP, Berne

Le diagnostic sûr de la mort est une condition préalable essentielle en médecine de transplantation, dans la mesure où celle-ci repose sur le prélèvement d'organes. Il revêt une importance éthique, juridique et politique considérable, car il doit garantir que nous traitons toujours les vivants comme des vivants et les morts comme des morts. Les directives de l'ASSM Diagnostic de la mort dans le contexte de la transplantation d'organes (mai 2011) sont en cours de révision et le projet de texte est en consultation jusqu'à fin février 2017.

Un diagnostic fiable de la mort exige, d'une part, un critère clair et universel pour établir la survenance du décès et, d'autre part, une méthode fiable pour constater la mort. Dans la loi sur la transplantation (LTx), le critère retenu pour constater la mort d'un être humain est la défaillance irréversible de toutes les fonctions du cerveau, y compris celles du tronc cérébral. Concernant les étapes cliniques du diagnostic de la mort, l'Ordonnance sur la transplantation renvoie aux chapitres correspondants des directives Diagnostic de la mort dans le contexte de la transplantation d'organes de l'Académie Suisse des Sciences Médicales (ASSM) du 24 mai 2011.

En juin 2015, le Parlement avait adopté une version révisée de la LTx. Elle entrera en vigueur dès que les adaptations nécessaires auront été réalisées dans les ordonnances. Le 21 octobre dernier, le DFI a mis en consultation les projets de texte en question; la consultation est ouverte jusqu'au 3 février 2017. Suite à la révision de la LTx, une révision des directives de l'ASSM s'est également imposée. La Commission Centrale d'Ethique a chargé une sous-commission ${ }^{1}$, dirigée par le Prof. Jürg Steiger de Bâle, de procéder aux adaptations nécessaires.

Une motion concernant l'attribution d'organes aux frontaliers est à l'origine de la révision de la LTx. Dans le cadre de la révision, des attentes importantes de la pratique ont été prises en considération, entre autres la question de savoir à partir de quel moment les proches doivent être impliqués, respectivement si les proches peuvent ou non consentir aux mesures médicales préliminaires. Les directives révisées traitent ces points essentiels en détail et proposent entre autres une visualisation (flowchart) du processus décisionnel (annexe B). Les éléments essentiels de la révision sont récapitulés à la page suivante.

Dès la phase d'élaboration, la sous-commission a fait appel à des experts, notamment des représentants des hôpitaux effectuant des prélèvements d'organes, des sociétés de discipline concernées et des soins, mais également des organisations de patients, et a tenu compte de leurs remarques. Le Sénat a adopté le projet de texte pour la consultation lors de sa séance du 16 novembre 2016. La consultation dure jusqu'à fin février 2017; les personnes et les organisations intéressées sont invitées à soumettre leurs commentaires.

Les points essentiels suivants sont restés inchangés dans les directives de l'ASSM:

- Les conditions du critère de la mort (signes cliniques);

- L'examen par deux médecins spécialistes (principe de surveillance mutuelle), pas d'intervalle de temps;

- Un examen technique complémentaire n'est nécessaire que lorsque les conditions requises pour l'examen clinique ne sont pas remplies (c'est-à-dire lorsque l'examen clinique des nerfs cérébraux est impossible ou que l'éventualité de facteurs potentiellement réversibles ne peut être exclue);

- Pas de prélèvement d'organes chez les nouveau-nés.

\section{Projet de texte pour la consultation et autres informations}

www.samw.ch $\rightarrow$ Ethique $\rightarrow$ Transplantation $\rightarrow$ Révision des directives 


\section{Les éléments essentiels des directives révisées}

A quel moment la question du don d'organes peut-elle être abordée avec les proches?

La séparation claire entre la décision d'interrompre un traitement vital (modification de l'objectif thérapeutique) et la décision de donner des organes doit être garantie. Même si les proches abordent, à un stade précoce, le sujet du don d'organes, le consentement au prélèvement et, le cas échéant, aux mesures médicales préliminaires ne peut être recherché qu'après avoir décidé d'interrompre les thérapies de maintien en vie. Les entretiens peuvent néanmoins être menés avant la constatation de la mort.

\section{Quelles conditions doivent être remplies avant de pouvoir établir un diagnostic de la mort?}

Sur la base de remarques issues de la pratique, les conditions cliniques devant être remplies avant de pouvoir procéder au diagnostic de la mort sont décrites en annexe $C$.

\section{Qui est habilité à constater la mort?}

Lévaluation clinique doit être effectuée par des médecins spécialistes bénéficiant d'une formation postgraduée et d'une expérience suffisante dans le domaine du diagnostic de la mort cérébrale. L'expérience est suf- fisante lorsqu'au moins un des deux médecins spécialistes chargés du diagnostic a établi, sous supervision, un diagnostic de mort cérébrale chez au moins cinq patients.

\section{Quelles mesures préliminaires peuvent être réalisées avant la mort?}

Conformément à la LTx, les mesures préliminaires peuvent être appliquées avant la mort du donneur, lorsqu'il y a consenti lui-même ou que ses proches y ont consenti, dans la mesure où ils n'ont pas connaissance d'un refus du donneur. Dans la nouvelle version des directives, ce processus décisionnel complexe est présenté dans un schéma (flowchart, annexe B). Les directives énumèrent, en outre, dans une liste négative (annexe $\mathrm{H}$ ), les mesures préliminaires qui ne peuvent être appliquées dans aucune situation, car elles ne sont pas indispensables à une transplantation réussie et comportent des risques plus que minimaux.

\section{Comment gérer les situations où des proches refusent} un prélèvement d'organes par conviction personnelle malgré l'existence d'une carte de donneur?

Les directives renvoient à la réglementation juridique qui précise que la volonté du défunt prime celle des proches. Elles recommandent toutefois à toutes les personnes concernées d'essayer de trouver un accord et, si nécessaire, d'avoir recours à un soutien éthique. 